\title{
Modelo matemático para a tomada de decisão para sistema predial de água não potável: descentralizado ou centralizado?
}

\author{
Mathematical model for decision-making for non-potable \\ water system of residential buildings: decentralised or \\ centralised?
}

\begin{abstract}
Christine Miranda Dias
Lúcia Helena de Oliveira

Fernando Akira Kurokawa

\section{Resumo}

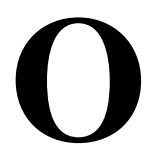

s sistemas prediais de água não potável podem ser do tipo descentralizado individual, descentralizado em grupo ou centralizado. Não há consenso sobre qual deles é o mais eficiente, uma vez que tanto os sistemas centralizados como os descentralizados possuem particularidades que os tornam interessantes ou não em aspectos sociais, econômicos e ambientais. Assim, o objetivo deste artigo é desenvolver a formulação de um modelo matemático para a tomada de decisão quanto ao tipo de sistema de água não potável e comparar os sistemas descentralizados com o sistema centralizado no período de 20 anos, com e sem o efeito escala. O modelo foi desenvolvido com base em uma abordagem matemática determinística, conhecida na literatura como programação inteira. Os diferenciais desse modelo é que ele permite determinar o custo total acumulado, o custo ao longo da vida útil e a quantidade de sistemas para atender a uma demanda específica. O modelo foi aplicado a um condomínio residencial hipotético com uma população mínima de 1.700 habitantes. Os resultados indicam que o sistema centralizado é mais viável do que os sistemas descentralizados em termos de custos de implantação, manutenção e de operação para a vida útil de 20 anos, com e sem o efeito escala.
\end{abstract}

Palavras-chave: Água não potável. Tomada de decisão. Sistema centralizado. Sistema descentralizado. Programação inteira.

\begin{abstract}
There are three types of non-potable water building systems: decentralised individual, decentralised or centralised. There is no consensus on which the most efficient type is, since both centralised and decentralised systems have peculiarities that make them either interesting or not in social, economic and environmental aspects. Thus, the aim of this article is to develop the formulation of a mathematical model for decision making regarding the type of non-potable water system and compare decentralised systems with centralised systems over a period of twenty years, with and without the scale effect. The model was developed based on a deterministic mathematical approach, known in the literature as Integer Programming. The difference of this model is that it allows to determine the total accumulated cost, the cost over life cycle and the number of systems necessary to meet a specific demand. The model was applied to a hypothetical residential condominium with a minimum population of 1700 residents. The results indicate that the centralised system is more advantageous than the individual systems in terms of costs of installation, maintenance and operation during a life cycle of twenty years with and without the scale effect.
\end{abstract}

${ }^{3}$ Fernando Akira Kurokawa

3Universidade de São Paulo São Paulo - SP - Brasil

Recebido em 22/03/19 Aceito em 22/05/19

Keywords: Non-potable water. Decision-making. Centralised system. Decentralised system. Integer programming.

DIAS, C. M.; OLIVEIRA, L. H. de; KUROKAWA, F. A. Modelo matemático para a tomada de decisão para sistema predial 385 de água não potável: descentralizado ou centralizado? Ambiente Construído, Porto Alegre, v. 20, n. 2, p. 385-400, abr./jun. 2020.

ISSN 1678-8621 Associação Nacional de Tecnologia do Ambiente Construído.

http:// dx. doi. org/ 10.1590/ s1678-86212020000200405 


\section{Introdução}

A tomada de decisão no que diz respeito ao uso de água não potável em edifícios deve englobar prioritariamente todos os riscos que envolvem sua utilização. Quanto aos custos desses sistemas, tão importante quanto considerar os de implantação é analisar as despesas associadas a sua operação e manutenção ao longo da vida útil.

As pesquisas relacionadas ao uso de água não potável se concentram na possibilidade de seu uso em edifícios (MANOUCHEHRI; KAGARI, 2017; COOK; SHARMAA; GURUNG, 2014), na avaliação durante a operação (CASTILHO; OLIVEIRA, 2018), na avaliação da aceitação pública desses sistemas (DOMENÉCH; SAURÍ, 2010) e nos riscos de contaminação da água potável (FERREIRA; OLIVEIRA, 2018; SCHEE, 2004, DIXON; BUTLER; FEWKES, 1999).

É usual no mercado brasileiro avaliar economicamente os sistemas prediais de água não potável com base somente nos custos de implantação ou com base nos custos do primeiro ano de operação, o que é um equívoco. Ocorre que, com o passar do tempo, os custos operacionais podem ter seus valores invertidos, passando de um sistema menos vantajoso para um mais vantajoso (DIAS, 2017). Por essa razão, é importante uma análise comparativa das opções durante a vida útil, quando do processo de escolha de um sistema mais viável economicamente. Outra questão que implica redução de custos é o efeito escala, quando ocorre o aumento da demanda de água não potável.

De acordo com Engin e Demir (2006), são quatro os fatores que influenciam o processo de tomada de decisão acerca da descentralização, especialmente no caso de pequenas comunidades, quais sejam: custos, flexibilidade no uso do terreno devido à menor ocupação física em comparação aos sistemas centralizados, manutenção e proteção ambiental.

A maioria dos tipos de tratamento favorece a opção por sistemas centralizados, uma vez que compreendem tecnologias mais conhecidas e proporcionam maior controle do insumo ofertado. Por outro lado, o custo de execução da rede de coleta de esgoto e de distribuição de água não potável favorece a descentralização devido à proximidade do ponto de geração de efluentes, do local de tratamento e do consumo da água não potável (GUO; ENGLEHARDT, 2015).

Durante o processo de tomada de decisão é fundamental identificar todos os objetivos a serem alcançados, além das consequências resultantes das possíveis opções (OLIVEIRA, 2004; GOLDBARG; LUNA, 2005).

Uma das abordagens para a tomada de decisão que pode ser empregada em problemas que envolvem distribuir recursos limitados entre atividades que vão competir por aquele recurso da melhor maneira possível é a Programação Inteira (PI). Trata-se de uma modelagem matemática que envolve fenômenos estáticos, também denominados determinísticos (ARENALES et al., 2007). A PI tem como base modelos e algoritmos que permitem criar um procedimento coerente e consistente para a tomada de decisão de problemas a serem solucionados (MARINS, 2011).

Enfim, entende-se que a escolha entre sistema de água não potável centralizado ou descentralizado requer uma análise criteriosa e complexa, visto que a decisão não depende somente de fatores que incidem de maneira isolada. É indispensável a avaliação sistêmica das necessidades, intervenções e consequências para correlacionar todas as variáveis com seus respectivos custos a fim de ponderar de maneira precisa as vantagens e desvantagens inerentes a cada sistema.

Nesse contexto, o objetivo deste artigo é desenvolver a formulação de um modelo matemático com base em PI que permita otimizar a tomada de decisão por meio das principais variáveis envolvidas na implantação, manutenção e operação de sistemas de tratamento de efluentes. Além disso, comparar ao longo da vida útil sistemas descentralizados, individuais e em grupo, com o sistema centralizado, e também sob a influência do efeito escala.

\section{Referencial teórico}

Nesta seção apresentam-se os tipos de sistemas prediais de água não potável e os fundamentos da PI.

\section{Sistemas prediais de água não potável}

O sistema predial de água não potável é um conjunto de tubulações, reservatórios, equipamentos e outros componentes destinados a coletar, armazenar, tratar e distribuir a água que não atende ao padrão de potabilidade (MARQUES; OLIVEIRA, 2014). 
Entre as vantagens da implantação de um sistema predial de água não potável encontra-se a redução da demanda de água potável e do volume de esgoto sanitário destinado ao sistema urbano de coleta. São apresentados a seguir os tipos de sistemas prediais de água não potável segundo a lógica de concentração de tratamento dos efluentes.

No sistema centralizado de água não potável, ilustrado na Figura 1, os efluentes originários de diversas edificações são coletados e transportados para um único local, a estação de tratamento de esgoto (ETE), onde parte do esgoto é tratada e distribuída às edificações para atividades com uso de água não potável, e o excedente é transportado para o sistema público de esgoto sanitário.

As alternativas para o sistema descentralizado são do tipo individual e em grupo. No sistema predial descentralizado individual de água não potável a coleta do efluente, o transporte, o tratamento e o uso da água não potável ocorrem em uma única residência ou edifício (UNITED..., 2004), conforme ilustrado na Figura 2.

No sistema descentralizado em grupo, a coleta de efluentes ocorre em mais de uma unidade e é direcionada a um local de tratamento, para então retornar à população como água não potável (UNITED..., 2004). Um exemplo de um sistema descentralizado em grupo é apresentado na Figura 3.

Hendrickson et al. (2015) salientam que em áreas urbanas densamente povoadas existem entraves geográficos e socioeconômicos desafiadores para os sistemas centralizados. Segundo os autores, esse tipo de sistema apresenta dificuldades relacionadas às restrições espaciais, o que impede a expansão das instalações com o crescimento da demanda, além de exigir redes de distribuição diferenciadas para a água potável e para a água não potável.

Em princípio, um sistema centralizado seria economicamente mais viável quando comparado a um conjunto de sistemas descentralizados individuais devido ao fato de o sistema centralizado ser capaz de atender a um grupo de edifícios sempre por meio de uma equipe capacitada. No caso de sistemas descentralizados individuais, isso não ocorre, uma vez que os gestores de cada edifício, em geral, não são capacitados.

Figura 1 - Sistema centralizado de água não potável

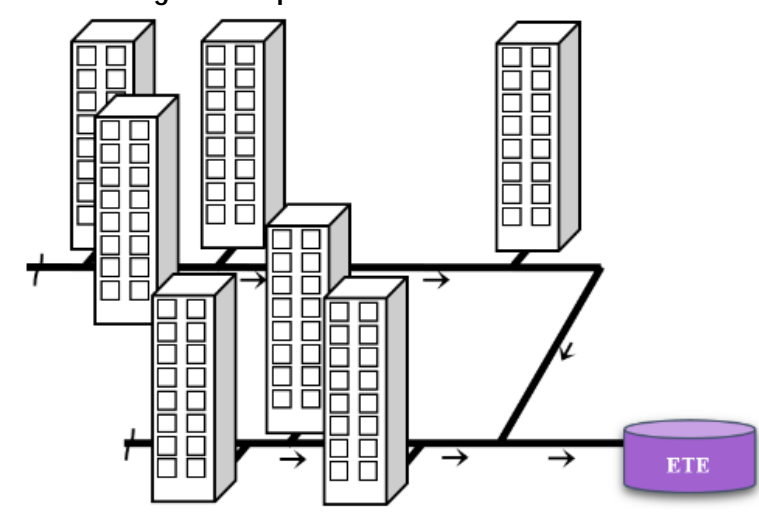

Fonte: Ferreira e Oliveira (2018).

Figura 2 - Sistemas descentralizados individuais de água não potável

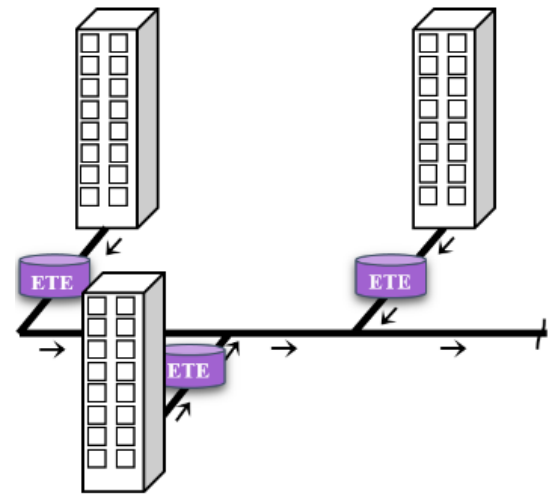

Fonte: Ferreira e Oliveira (2018). 
Figura 3 - Sistema descentralizado em grupo de água não potável

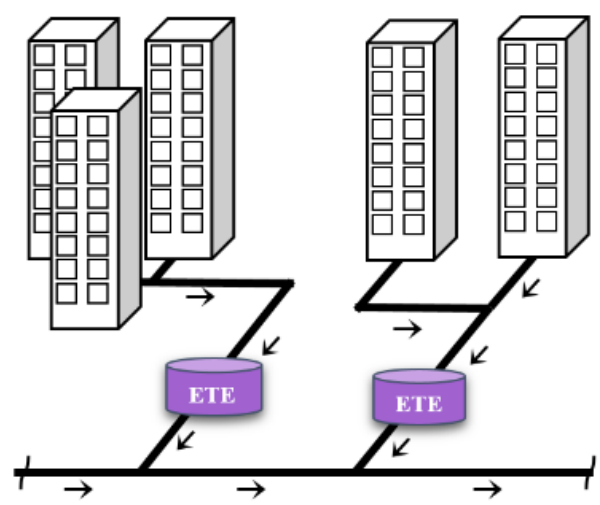

Fonte: Ferreira e Oliveira (2018).

Por outro lado, nos sistemas descentralizados a coleta, o tratamento e o transporte dos efluentes gerados pela população ocorrem próximos aos locais de sua produção. Asano et al. (2007) ressaltam que o grau de descentralização pode variar desde uma unidade habitacional, um edifício ou até um bairro. Afferden et al. (2010) afirmam que, para terem bom desempenho, os sistemas descentralizados devem ser controlados por uma administração externa. Nesse aspecto, Cohim et al. (2009) acrescentam que tanto os sistemas centralizados quanto os descentralizados devem ser operados por uma autoridade central capacitada, a fim de minimizar os riscos à saúde dos usuários e de aumentar a credibilidade do uso de água não potável.

Guest et al. (2009) e Libralato, Ghirardini e Avezzù (2012) defendem a coexistência de sistemas centralizados e descentralizados, com vários níveis de aplicabilidade em diferentes regiões, sendo que, em princípio, a tomada de decisão por um tipo de sistema não pode excluir a implantação do outro. Para os autores, essa abordagem seria a mais realista nas condições atuais das cidades, especialmente no caso de grandes conjuntos de edificações, tais como centros comerciais, hospitais, aeroportos ou em novas áreas de desenvolvimento, onde o uso de água não potável pode ser efetivamente planejado.

Com base no que foi exposto, percebe-se que não há consenso acerca de qual sistema, centralizado ou descentralizado, seja o mais adequado para edifícios residenciais. Cada um deles apresenta vantagens particulares que devem ser avaliadas considerando tanto aspectos diretos, como, por exemplo, infraestrutura e custos envolvidos, quanto indiretos, como características regionais e impactos ambientais.

\section{Fundamentos teóricos da Programação Inteira}

A PI é um problema de otimização que apresenta os seguintes atributos (COLIN, 2007):

(a) um conjunto de variáveis manipuláveis, chamadas de variáveis de decisão do problema, no procedimento de busca pelo resultado ótimo;

(b) a função objetivo é uma função linear escrita em termos dessas variáveis de decisão; e

(c) os valores assumidos pelas variáveis de decisão devem satisfazer um conjunto de restrições, que compõem a região de soluções viáveis do problema.

A PI é classificada em três tipos:

(d) programação inteira pura: todas as variáveis são inteiros genéricos;

(e) programação inteira mista: parte das variáveis é de inteiros e parte é de variáveis contínuas; e

(f) programação inteira com variáveis 0-1: todas as variáveis assumem valores 0 ou 1.

A finalidade da PI é determinar a solução ótima para problemas que sejam representados por uma expressão linear, denominada função objetivo, que será maximizada ou minimizada, respeitando-se um sistema linear de igualdades ou desigualdades, que recebem o nome de restrições do modelo. Essas restrições determinam uma região chamada conjunto viável, aquela que maximiza ou minimiza a função objetivo, sendo a melhor das soluções viáveis existentes a solução ótima (MARINS, 2011). Desse modo, a estrutura básica de um modelo de PI pode ser descrita conforme a Equação 1 (GARFINKEL; NEMHAUSER, 1972). 
(MAX) ou (MIN) $Z=c_{1} X_{1}+c_{2} X_{2}+\cdots+c_{n} X_{n}$

sujeita às $\left\{\begin{array}{c}a_{11} X_{1}+a_{12} X_{2}+\cdots+a_{1 n} X_{n} \leq b_{1} \\ a_{21} X_{1}+a_{22} X_{2}+\cdots+a_{2 n} X_{n} \leq b_{2} \\ \vdots \\ a_{m 1} X_{1}+a_{m 2} X_{2}+\cdots+a_{m n} X_{n} \leq b_{m} \\ X_{i} \in\{0,1\} \text { ou } X_{i} \in \mathbb{Z}_{+}\end{array}\right.$

As constantes $a_{i j}$, $b_{i}$ e $c_{j}$ são os parâmetros do modelo, particularmente denominados de coeficientes das restrições, constantes do lado direito e coeficientes da função objetivo respectivamente.

\section{Método}

O que norteou o desenvolvimento deste trabalho foi a proposição de um modelo que permita ao profissional decidir pela melhor opção de sistema de água não potável para determinado tipo de tratamento de água residuária em uma edificação. Nesse contexto, a premissa necessária para o desenvolvimento da formulação do modelo foi a realização de uma pesquisa bibliográfica para coletar dados de modo a caracterizar e a comparar tanto os sistemas centralizados e descentralizados quanto os tipos de tratamento levantados.

Com os dados coletados desenvolveu-se a formulação do modelo matemático para a tomada de decisão com base na PI. A solução do modelo foi obtida com o auxílio do software LINDO (Linear, Interactive, and Discrete Optimizer). Na Figura 4 estão ilustradas as etapas da pesquisa, as quais são descritas a seguir.

\section{Levantamento de dados}

Inicialmente foi realizada uma pesquisa bibliográfica sobre o tema para levantar dados relativos às variáveis qualitativas e quantitativas que envolvem o processo de tomada de decisão quanto ao tipo de sistema de tratamento de efluentes.

\section{Estabelecimento das variáveis}

As variáveis mais recorrentes e consideradas de maior importância para a comparação entre os tipos de sistemas são custo de implantação, custo de operação e custo de manutenção.

\section{Figura 4 - Etapas da pesquisa}

\section{Levantamento de dados}

- Estabelecimento das variáveis a serem inseridas no modelo

- Levantamento quantitativo das variáveis

\section{Formulação e resolução do modelo matemático}

- Definição da função objetivo e das restrições

- Escolha do software para a resolução do modelo

\section{Estudo de caso: condomínio residencial hipotético}


Ressalta-se que o custo de implantação inclui as despesas de aquisição dos sistemas e das obras civis necessárias. O custo de manutenção engloba a análise físico-química da água não potável produzida, a reposição de produtos químicos, a remoção do lodo e a limpeza dos equipamentos, componentes e reservatórios (DELL'UOMO, 2014; DOMINGUES et al., 2016). O custo de operação, obtido na literatura, engloba o consumo de energia elétrica para o tratamento da água residuária. Os consumos de energia elétrica relacionados à operação das bombas para o recalque da água não potável do reservatório inferior para o superior não foram contabilizados.

As despesas com lubrificações, ajustes e reposições de componentes e acessórios, bem como com a manutenção corretiva para a substituição de equipamentos, também não foram consideradas no cálculo dos custos de manutenção. Além disso, não foram encontrados na literatura dados relativos à taxa real de reajuste dos custos de manutenção dos diferentes tipos de tratamento de água não potável. Por essa razão, considerouse que os custos de manutenção dos sistemas apresentam um aumento de custo simbólico de 1\% ao ano.

Para os sistemas hidrossanitários, em que os sistemas prediais de água não potável estão inseridos, a vida útil de projeto para o nível de desempenho mínimo, estabelecida pela NBR 15575 (ABNT, 2013) é de 20 anos, período que foi considerado no modelo. No entanto, com o intuito de entender melhor o comportamento dos sistemas, a aplicação do modelo e a análise dos resultados foram realizadas para cada quinquênio de operação.

\section{Levantamento quantitativo das variáveis}

Após o estabelecimento do conjunto de variáveis custos de implantação, de operação e de manutenção, foram coletados dados quantitativos a serem utilizados na aplicação do modelo. Os valores dessas variáveis foram obtidos das seguintes referências bibliográficas: Domingues et al. (2016), Dell’Uomo (2014), Reami e Coraucci Filho (2010) e Von Sperling (2005).

\section{Formulação e resolução do modelo matemático}

A partir do estabelecimento das variáveis e dos dados coletados foi possível desenvolver uma formulação do modelo matemático para a tomada de decisão com base nos princípios da PI.

\section{Definição da função objetivo e das restrições}

Para definir a função objetivo e atribuir as restrições do modelo de PI, primeiramente é preciso saber quais são as variáveis de decisão. Para esse problema, as variáveis de decisão, denominadas de $X_{i j}$, são aquelas que representam a possibilidade ou a impossibilidade de instalação de determinado sistema para satisfazer uma demanda específica, em que $i$ representa as $n$ opções com tipos de sistemas disponíveis, e $j$ representa os $m$ tipos de custos relativos ao tipo de sistema da opção $i$. Em função dessas variáveis de decisão, é definida a função objetivo, $Z$, e atribuídas as restrições do modelo, de acordo com a Equação 2.

(MIN) $Z=\sum_{i=1}^{n} \sum_{j=1}^{m} A_{i j} X_{i j}$

sujeita às $\left\{\begin{array}{cc}\sum_{i=1}^{n} X_{i j}=1, & j=1,2, \ldots, m \\ X_{i j+1}-X_{i j} \leq 0, & i=1,2, \ldots, n \text { e } j=1,2, \ldots, m-1 \\ X_{i j} \in\{0,1\} & \forall i, j\end{array}\right.$

Os coeficientes $A_{i j}$ da função objetivo (Z) correspondem aos custos de implantação - $A_{i 1}$, de manutenção $A_{i 2}$, de operação - $A_{i 3}$, etc., dos sistemas da opção $i$. É importante destacar que os valores de $i$ e $j$ se modificam conforme a quantidade de opções disponíveis ao projetista e de custos necessários para realizar a comparação. A função objetivo $(Z)$ está minimizada, pois se deseja encontrar o menor custo total, e está sujeita às restrições.

Como o modelo foi desenvolvido com base nos princípios de PI, em que todas variáveis são do tipo 0 e 1 , as $m$ primeiras restrições representam grupos de alternativas mutuamente exclusivas, ou seja, em cada restrição apenas uma variável deve ser igual a $1\left(X_{k j}=1\right.$ em que $k$ pode ser 1 ou $2 \ldots$ ou $\left.n\right)$, e as variáveis que representam os custos das outras opções devem ser iguais a zero $\left(X_{l j}=0, l \neq k\right)$.

As $n+m$ inequações referem-se às decisões contingentes, ou seja, que podem ou não ocorrer, mas que dependem dos resultados de decisões anteriores. Por exemplo, qualquer que seja a resposta para a variável $X_{11}$, consequentemente as variáveis $X_{12}, X_{13}, \ldots, X_{1 m}$ terão os mesmos resultados. O mesmo vale para as outras variáveis do modelo. 
Essas restrições significam que o modelo nunca associará os custos de opções diferentes, como o custo de implantação da opção 1 com o custo de manutenção da opção 2, com o custo de operação da opção 3, e assim por diante, porque a coexistência desses custos não seria possível em uma situação real.

Assim, esse modelo tem por objetivo indicar não apenas qual o menor custo total acumulado entre as diferentes opções de distribuição dos sistemas, mas também quantos e quais sistemas são necessários para atender à demanda de uma população com base nos dados obtidos nas referências consultadas.

\section{Escolha do software para a resolução do modelo}

Para determinar a solução ótima do modelo foi utilizado o software LINDO, versão 6.1, desenvolvido pela LINDO Systems Inc. Esse software é capaz de resolver problemas de programação linear, inteira e quadrática. Apesar de existirem no mercado outros softwares que solucionem problemas de PI, o LINDO foi escolhido devido à simplicidade de sua interface para a inserção dos dados e à agilidade na resolução dos modelos. Além disso, as soluções obtidas são apresentadas de forma organizada e detalhada.

\section{Estudo de caso: condomínio residencial hipotético}

O modelo de tomada de decisão foi aplicado a um condomínio residencial hipotético. Esse condomínio é composto de dez edifícios residenciais com catorze pavimentos tipo, quatro apartamentos por andar e capacidade de abrigar uma população média de 170 pessoas, totalizando ao menos 1.700 moradores.

Os dados utilizados para a formulação do modelo são apresentados na Tabela 1 e baseiam-se nas características dos sistemas detalhados em Reami e Coraucci Filho (2010), Dell’Uomo (2014) e Domingues et al. (2016) para o primeiro ano de operação dos sistemas. As informações ausentes foram adaptadas de Von Sperling (2005).

A partir das máximas populações atendidas são possíveis as seguintes opções para distribuir os sistemas a fim de satisfazer a demanda desse condomínio:

(a) opção 1: dez sistemas descentralizados individuais capazes de suprir a demanda de até 1.700 habitantes;

(b) opção 2: cinco sistemas descentralizados em grupo capazes de suprir a demanda de até 1.800 habitantes; e

(c) opção 3: um sistema centralizado capaz de suprir a demanda de até 1.719 habitantes.

\section{Resultados e discussões}

Nesta seção são apresentados os resultados e as discussões para o modelo de tomada de decisão desenvolvido. Esse modelo tem o objetivo de indicar quantos e quais sistemas devem ser implantados a fim de satisfazer a demanda do condomínio hipotético, com o menor custo total ao longo da vida útil de 20 anos do sistema. Para esse caso foram considerados os custos de implantação, manutenção e operação.

Tabela 1 - População, custos e consumo de energia de cada sistema indicado na literatura no primeiro ano de operação

\begin{tabular}{c|l|c|c|c|c}
\hline Sistema & \multicolumn{1}{|c|}{$\begin{array}{c}\text { Tipo de } \\
\text { tratamento }\end{array}$} & $\begin{array}{c}\text { Máxima } \\
\text { população } \\
\text { atendida }\end{array}$ & $\begin{array}{c}\text { Custo de } \\
\text { implantação } \\
\text { (US\$) }\end{array}$ & $\begin{array}{c}\text { Custo de } \\
\text { manutenção } \\
\text { (US\$/ano) }\end{array}$ & $\begin{array}{c}\text { Consumo de } \\
\text { energia } \\
\text { (kWh/mês) }\end{array}$ \\
\hline 1 & $\begin{array}{l}\text { Disco biológico } \\
\text { rotativo (DBR) }^{[1]}\end{array}$ & $170^{[1]}$ & $43.486^{[1]}$ & $16.780^{[1]}$ & $0^{[1]}$ \\
\hline 2 & Físico-químico $^{[2]}$ & $360^{[2]}$ & $60.642^{[2]}$ & $23.824^{[2]}$ & $1.940^{[2]}$ \\
\hline 3 & $\begin{array}{l}\text { Reator UASB com } \\
\text { filtro biológico } \\
\text { aerado submerso }^{[3]}\end{array}$ & $1.719^{[3]}$ & $305.229^{[3]}$ & $7.640^{[4]}$ & $2.865^{[4]}$ \\
\hline
\end{tabular}

Fonte: elaborada a partir de ${ }^{[1]}$ Domingues et al. (2016), ${ }^{[2]}$ Dell'Uomo (2014), ${ }^{[3]}$ Reami e Coraucci Filho (2010) e ${ }^{[4]}$ Von Sperling (2005). 


\section{Modelo sem a consideração do efeito escala}

Considerando-se os custos de implantação, manutenção e operação, a partir da Equação 2 chega-se ao modelo de PI, para cada quinquênio de operação dos sistemas e sem considerar o efeito escala, expresso pela Equação 3.

(MIN) $Z=A_{11} X_{11}+A_{12} X_{12}+A_{13} X_{13}+A_{21} X_{21}+A_{22} X_{22}+A_{23} X_{23}+A_{31} X_{31}+A_{32} X_{32}+A_{33} X_{33}$

$$
\text { sujeita às }\left\{\begin{array}{c}
X_{11}+X_{21}+X_{31}=1 \\
X_{12}+X_{22}+X_{32}=1 \\
X_{13}+X_{23}+X_{33}=1 \\
X_{12}-X_{11} \leq 0 \\
X_{13}-X_{12} \leq 0 \\
X_{22}-X_{21} \leq 0 \\
X_{23}-X_{22} \leq 0 \\
X_{32}-X_{31} \leq 0 \\
X_{33}-X_{32} \leq 0 \\
X_{i j} \in\{0,1\}
\end{array}\right.
$$

Os coeficientes $A_{i 1}$ correspondem aos custos de implantação da opção $i$. Para os custos de manutenção e operação é preciso levar contar a vida útil dos sistemas. Desse modo, os coeficientes $A_{i 2}$, referentes aos custos de manutenção acumulados ao longo do tempo de análise, são calculados a partir da Equação 4.

$A_{i 2}=C_{i 2}+C_{i 2} \times(1+t)+\cdots+C_{i 2} \times(1+t)^{v-1}$

Onde:

$C_{i 2}$ o custo de manutenção da opção $i$ no primeiro ano de operação, que equivale ao somatório do custo de manutenção de cada tipo de sistema englobado na opção $i$;

$t$ a taxa de aumento anual no custo de manutenção definida para cada opção $i$; e

$v$ a vida útil, em anos, determinada para realizar a análise.

Os coeficientes $A_{i 3}$, que representam os custos de operação acumulados ao longo do tempo de análise, são calculados a partir da Equação 5.

$A_{i 3}=C_{i 3}+C_{i 3} \times(1+s)+\cdots+C_{i 3} \times(1+s)^{v-1}$

Onde:

$A_{i 3}$ o custo de operação da opção $i$ no primeiro ano de operação, igual ao somatório do custo de operação de cada tipo de sistema englobado na opção $i$;

$s$ a taxa de reajuste anual na tarifa de energia elétrica, que, neste trabalho, é de 10,35\% ao ano; e

$v$ a vida útil, em anos, determinada para realizar a análise.

Como as opções 1 e 2 possuem mais de um sistema a serem instalados, os custos totais de implantação, manutenção e operação foram calculados, respectivamente, multiplicando-se os custos de implantação, manutenção e operação de um sistema pela quantidade a ser implantada no condomínio. Na Tabela 2 estão apresentados os custos de cada opção para o primeiro ano de operação.

Tabela 2 - Custos de implantação, manutenção e operação de cada opção no primeiro ano de operação

\begin{tabular}{c|l|c|c|c|c}
\hline Opção & \multicolumn{1}{|c|}{ Tipo de sistema } & $\begin{array}{c}\text { Quantidade } \\
\text { de sistemas }\end{array}$ & $\begin{array}{c}\text { Custo de } \\
\text { implantação } \\
\text { (US\$) }\end{array}$ & $\begin{array}{c}\text { Custo de } \\
\text { manutenção } \\
\text { (US\$/ano) }\end{array}$ & $\begin{array}{c}\text { Custo de } \\
\text { operação } \\
\text { (US\$/ano) }\end{array}$ \\
\hline 1 & $\begin{array}{l}\text { Disco biológico } \\
\text { rotativo (DBR) }\end{array}{ }^{[1]}$ & 10 & $434.860^{[1]}$ & $167.800^{[1]}$ & $0^{[1]}$ \\
\hline 2 & Físico-químico $^{[2]}$ & 5 & $303.210^{[2]}$ & $119.120^{[2]}$ & $3.280^{[2]}$ \\
\hline 3 & $\begin{array}{l}\text { Reator UASB com } \\
\text { filtro biológico aerado } \\
\text { submerso [3] }\end{array}$ & 1 & $305.229^{[3]}$ & $7.640^{[4]}$ & $5.809^{[4]}$ \\
\hline
\end{tabular}

Fonte: elaborada a partir de ${ }^{[1]}$ Domingues et al. (2016), [2] Dell'Uomo (2014), ${ }^{[3]}$ Reami e Coraucci Filho (2010) e ${ }^{[4]}$ Von Sperling (2005). 
Considerando-se que os custos de manutenção dos sistemas têm taxa de reajuste igual a 1\% ao ano, na Tabela 3 estão apresentados os custos de manutenção acumulados de cada opção em cada quinquênio de análise, calculados de acordo com a Equação 4.

Na Tabela 4 estão mostrados os custos de operação acumulados $\left(A_{i 3}\right)$ das opções em cada quinquênio de análise, calculados de acordo com a Equação 5.

Assim, com base nos dados das Tabelas 2, 3 e 4, formula-se para cada quinquênio o modelo que permite otimizar a tomada de decisão. Por exemplo, o modelo para o $20^{\circ}$ ano de operação dos sistemas é dado pela Equação 6:

(MIN) $Z=434.860 X_{11}+3.674,820 X_{12}+0 X_{131}+303.210 X_{21}+2.608,728 X_{22}+195.494 X_{23}+$ $305.229 X_{31}+167.316 X_{32}+346.228 X_{33}$

sujeita às $\left\{\begin{array}{c}X_{11}+X_{21}+X_{31}=1 \\ X_{12}+X_{22}+X_{32}=1 \\ X_{13}+X_{23}+X_{33}=1 \\ X_{12}-X_{11} \leq 0 \\ X_{13}-X_{12} \leq 0 \\ X_{22}-X_{21} \leq 0 \\ X_{23}-X_{22} \leq 0 \\ X_{32}-X_{31} \leq 0 \\ X_{33}-X_{32} \leq 0 \\ X_{i j} \in\{0,1\}\end{array}\right.$

Nota-se que as variáveis $X_{k j}=1(k=1$ ou $k=2$ ou $k=3)$ significam a possibilidade de instalar os sistemas de uma das três opções disponíveis, considerando todos os custos necessários, enquanto as variáveis $X_{l j}=0, l \neq k$ representam a impossibilidade de instalação devido ao fato de a outra opção apresentar um custo total acumulado menor. De maneira análoga, foram obtidos os modelos para o 5o, 10ํe 15oa ano de operação.

Ressalta-se que o período de análise quinquenal considerado nesse modelo pode ser qualquer outro, como, por exemplo, anual.

\section{Modelo com a consideração do efeito escala}

O custo de manutenção do sistema centralizado, opção 3, foi extraído de Von Sperling (2005), que “[...] aplicou os custos per capita considerando as faixas populacionais típicas de utilização de cada sistema de tratamento [...]”.

De acordo com Piveli (2004), a população atendida pelo reator UASB em associação com o filtro biológico aerado submerso está entre 20 e 200 mil habitantes. Desse modo, subentende-se que o efeito escala da elevada população está implícito no custo de manutenção de Von Sperling (2005), utilizado na opção 3. Para os sistemas descentralizados das opções 1 e 2 é considerada a influência do efeito escala com a inserção de sistemas somente para o custo de manutenção.

Tabela 3 - Custos de manutenção acumulados das opções para os quinquênios de análise

\begin{tabular}{c|c|c|c|c|c}
\hline \multirow{2}{*}{ Opção } & \multicolumn{5}{|c}{ Custo de manutenção acumulado - $\boldsymbol{A}_{\boldsymbol{i} 2}$ (US\$) } \\
\cline { 2 - 6 } & $\mathbf{1 0}$ ano & $\mathbf{5 0}$ ano & $\mathbf{1 0}$ ano & $\mathbf{1 5}$ ano & $\mathbf{2 0}$ ano \\
\hline 1 & 167.800 & 855.780 & $1.753,510$ & $2.693,190$ & $3.674,820$ \\
2 & 119.120 & 607.512 & $1.244,804$ & $1.911,876$ & $2.608,728$ \\
3 & 7.640 & 38.964 & 79.838 & 122.622 & 167.316 \\
\hline
\end{tabular}

Tabela 4 - Custos de operação acumulados das opções para os quinquênios de análise

\begin{tabular}{c|c|c|c|c|c}
\hline \multirow{2}{*}{ Opção } & \multicolumn{5}{|c}{ Custo de operação acumulado $-\boldsymbol{A}_{\boldsymbol{i 3}}$ (US\$) } \\
\cline { 2 - 6 } & $\mathbf{1 0}$ ano & $\mathbf{5 0}$ ano & $\mathbf{1 0}$ ano & $\mathbf{1 5}$ - ano & $\mathbf{2 0}$ ano \\
\hline 1 & 0 & 0 & 0 & 0 & 0 \\
2 & 656 & 4.033 & 10.632 & 21.430 & 39.099 \\
3 & 5.809 & 35.712 & 94.149 & 189.767 & 346.228 \\
\hline
\end{tabular}


Além disso, não foi encontrado na literatura um valor exato para o efeito escala que ocorre com a inserção de novas unidades de tratamento em um projeto, pois os custos de manutenção dependem principalmente das empresas especializadas em gerenciar sistemas. Todavia, em pesquisa para analisar as vantagens da descentralização de sistemas de tratamento de esgotos no Distrito Federal, Brostel e Harada (1997) apresentam dados que permitiram o cálculo do efeito escala com o acréscimo de sistemas no projeto.

Na Tabela 5 está apresentado o efeito escala médio, em porcentagem, sobre o custo de manutenção dos sistemas descentralizados, o qual foi obtido por meio de valores extraídos do estudo de Brostel e Harada (1997).

Por exemplo, o sistema DBR apresenta um custo de manutenção equivalente a US\$ 16.780 quando instalado somente um sistema. Para as condições da opção 1, na qual deverão ser instalados dez sistemas, o custo de manutenção de cada um será em torno de 21,13\% do custo total, caso fosse instalado isoladamente, ou seja, aproximadamente US\$ 3.5448. Portanto, o custo necessário à manutenção de dez sistemas será US\$ 35.448, arredondando-se os valores.

Assim, para as opções sugeridas, o custo de manutenção unitário de cada sistema foi recalculado considerando a influência do efeito escala com o aumento da quantidade de equipamentos instalados no condomínio, conforme apresentado na Tabela 6.

Considerando o efeito escala juntamente com a taxa de reajuste anual de $1 \%$, os custos de manutenção acumulados das três opções, em cada quinquênio de análise, foram recalculados e estão apresentados na Tabela 7.

Tabela 5 - Efeito escala médio no custo de manutenção de sistemas descentralizados

\begin{tabular}{c|c}
\hline $\begin{array}{c}\text { Quantidade de sistemas } \\
\text { implantados } \\
\text { (unidade) }\end{array}$ & $\begin{array}{c}\text { Efeito escala médio no } \\
\text { custo de manutenção } \\
\text { (\%) }\end{array}$ \\
\hline 1 & 100,00 \\
2 & 65,70 \\
3 & 47,81 \\
4 & 41,52 \\
5 & 33,79 \\
6 & 27,73 \\
7 & 25,50 \\
8 & 23,74 \\
9 & 22,31 \\
10 & 21,13 \\
\hline
\end{tabular}

Fonte: elaborada a partir de Brostel e Harada (1997).

Tabela 6 - Custos de manutenção unitários dos sistemas considerando o efeito escala

\begin{tabular}{|c|c|c|c|c|c|}
\hline \multirow{2}{*}{ Opção } & \multirow{2}{*}{$\begin{array}{l}\text { Quantidade } \\
\text { de sistemas }\end{array}$} & \multirow{2}{*}{$\begin{array}{l}\text { Efeito escala } \\
\text { no custo de } \\
\text { manutenção }\end{array}$} & \multicolumn{2}{|c|}{$\begin{array}{c}\text { Custo de manutenção } \\
\text { unitário (US\$/ano) }\end{array}$} & \multirow{2}{*}{$\begin{array}{c}\text { Custo de } \\
\text { manutenção } \\
\text { total das opções } \\
\text { (US\$/ano) } \\
\end{array}$} \\
\hline & & & $\begin{array}{l}\text { Sem efeito } \\
\text { escala }\end{array}$ & $\begin{array}{c}\text { Com efeito } \\
\text { escala }\end{array}$ & \\
\hline 1 & 10 & $21,13 \%$ & 16.780 & 3.545 & 35.448 \\
\hline 2 & 5 & $33,79 \%$ & 23.824 & 8.050 & 40.249 \\
\hline 3 & 1 & $100,00 \%$ & 7.640 & 7.640 & 7.640 \\
\hline
\end{tabular}

Tabela 7 - Custos de manutenção acumulados considerando o efeito escala

\begin{tabular}{c|c|c|c|c|c}
\hline \multirow{2}{*}{ Opção } & \multicolumn{5}{|c}{ Custo de manutenção acumulado (US\$) } \\
\cline { 2 - 6 } & $\mathbf{1 0}$ ano & $\mathbf{5 0}$ ano & $\mathbf{1 0}$ ano & $\mathbf{1 5}$ ano & 20\% ano \\
\hline 1 & 35.448 & 180.785 & 370.433 & 568.943 & 776.314 \\
2 & 40.249 & 205.270 & 420.603 & 645.998 & 881.455 \\
3 & 7.640 & 38.964 & 79.838 & 122.622 & 167.316 \\
\hline
\end{tabular}


Com os novos valores dos custos de manutenção acumulados (Tabela 7) formulou-se o modelo que permite otimizar a tomada de decisão para cada quinquênio considerando o efeito escala. Assim, no $20^{\circ}$ ano de operação dos sistemas, o modelo é dado pela Equação 7:

(MIN) $Z=434.860 X_{11}+776.314 X_{12}+0 X_{13}+303.210 X_{21}+881.455 X_{22}+195.494 X_{23}+$ $305.229 X_{31}+167.316 X_{32}+346.228 X_{33}$

sujeita às $\left\{\begin{array}{c}X_{11}+X_{21}+X_{31}=1 \\ X_{12}+X_{22}+X_{32}=1 \\ X_{13}+X_{23}+X_{33}=1 \\ X_{12}-X_{11} \leq 0 \\ X_{13}-X_{12} \leq 0 \\ X_{22}-X_{21} \leq 0 \\ X_{23}-X_{22} \leq 0 \\ X_{32}-X_{31} \leq 0 \\ X_{33}-X_{32} \leq 0 \\ X_{i j} \in\{0,1\}\end{array}\right.$

De maneira análoga, foram obtidas as formulações do modelo para o 5oㅗ $10^{\circ}$ e $15^{\circ}$ ano de operação.

\section{Discussões}

Para comparar os custos totais de sistemas descentralizados individuais, sistemas descentralizados em grupo e sistema centralizado, com e sem o efeito escala, que podem ser implantados nesse condomínio hipotético, foram realizadas as simulações para cada quinquênio do período de análise de 20 anos.

Por meio das formulações do modelo, os dados foram inseridos no software LINDO, com o intuito de obter a solução ótima para cada quinquênio de operação. Na Figura 5 está ilustrada a solução obtida para o modelo considerando o $20^{\circ}$ ano de operação dos sistemas e sem o efeito escala.

A informação destacada em vermelho na Figura 5 indica que o resultado da função objetivo é igual a US\$ 818.773, que é o valor ótimo do modelo no $20^{\circ}$ ano de operação. Esse valor representa o menor custo total entre as três opções disponíveis; todavia, para saber quantos sistemas são necessários, recorre-se à coluna value.

Observa-se que os resultados das variáveis $X_{11}, X_{12}, X_{13}, X_{21}, X_{22}$ e $X_{23}$ foram todos iguais a 0 , enquanto os resultados das variáveis $X_{31}, X_{32}$ e $X_{33}$ foram iguais a 1 . Isso significa que a opção 3 , representada pela implantação de um sistema centralizado, apresenta o menor custo total entre as opções disponíveis. Na Tabela 8 está apresentada a síntese dos resultados obtidos para cada quinquênio sem considerar o efeito escala.

Observa-se na Tabela 8 que a opção 3, que consiste na implantação de um sistema centralizado, apresenta o menor custo total durante o período de vida útil das opções comparadas. Na Figura 6 estão ilustrados os custos totais acumulados ao longo da vida útil de cada opção.

Figura 5 - Resultados da solução ótima sem o efeito escala do modelo fornecidos pelo software LINDO OBJECTIVE FUNCTION VALUE

\begin{tabular}{|cr|r|}
\hline 1$)$ & 818773.0 & \\
\hline VARIABLE & VALUE & REDUCED COST \\
X11 & 0.000000 & 43486.000000 \\
X12 & 0.000000 & 367482.000000 \\
X13 & 0.000000 & 0.000000 \\
X21 & 0.000000 & 60642.000000 \\
X22 & 0.000000 & 521746.000000 \\
X23 & 0.000000 & 39099.000000 \\
X31 & 1.000000 & 305229.000000 \\
X32 & 1.000000 & 167316.000000 \\
X33 & 1.000000 & 346228.000000 \\
\hline
\end{tabular}


Tabela 8 - Síntese dos resultados obtidos sem a consideração do efeito escala

\begin{tabular}{|c|c|c|c|c|c|}
\hline $\begin{array}{l}\text { Ano de } \\
\text { operação }\end{array}$ & $\begin{array}{c}\text { Resultado da } \\
\text { função objetivo } \\
\text { (US\$) }\end{array}$ & $\begin{array}{l}\text { Quantidade } \\
\text { de sistemas }\end{array}$ & Opção & $\begin{array}{l}\text { Tipo de } \\
\text { sistema }\end{array}$ & Tipo de tratamento \\
\hline $1^{\mathrm{o}}$ & 318.678 & 1 & 3 & Centralizado & $\begin{array}{l}\text { Reator UASB com filtro } \\
\text { biológico aerado submerso }\end{array}$ \\
\hline 50 & 379.905 & 1 & 3 & Centralizado & $\begin{array}{l}\text { Reator UASB com filtro } \\
\text { biológico aerado submerso }\end{array}$ \\
\hline $10^{\circ}$ & 479.216 & 1 & 3 & Centralizado & $\begin{array}{l}\text { Reator UASB com filtro } \\
\text { biológico aerado submerso }\end{array}$ \\
\hline $15^{\circ}$ & 617.618 & 1 & 3 & Centralizado & $\begin{array}{l}\text { Reator UASB com filtro } \\
\text { biológico aerado submerso }\end{array}$ \\
\hline $20^{\circ}$ & 818.773 & 1 & 3 & Centralizado & $\begin{array}{l}\text { Reator UASB com filtro } \\
\text { biológico aerado submerso }\end{array}$ \\
\hline
\end{tabular}

Figura 6 - Custo total acumulado sem o efeito escala durante a vida útil das três opções disponíveis para o condomínio com 1.700 habitantes

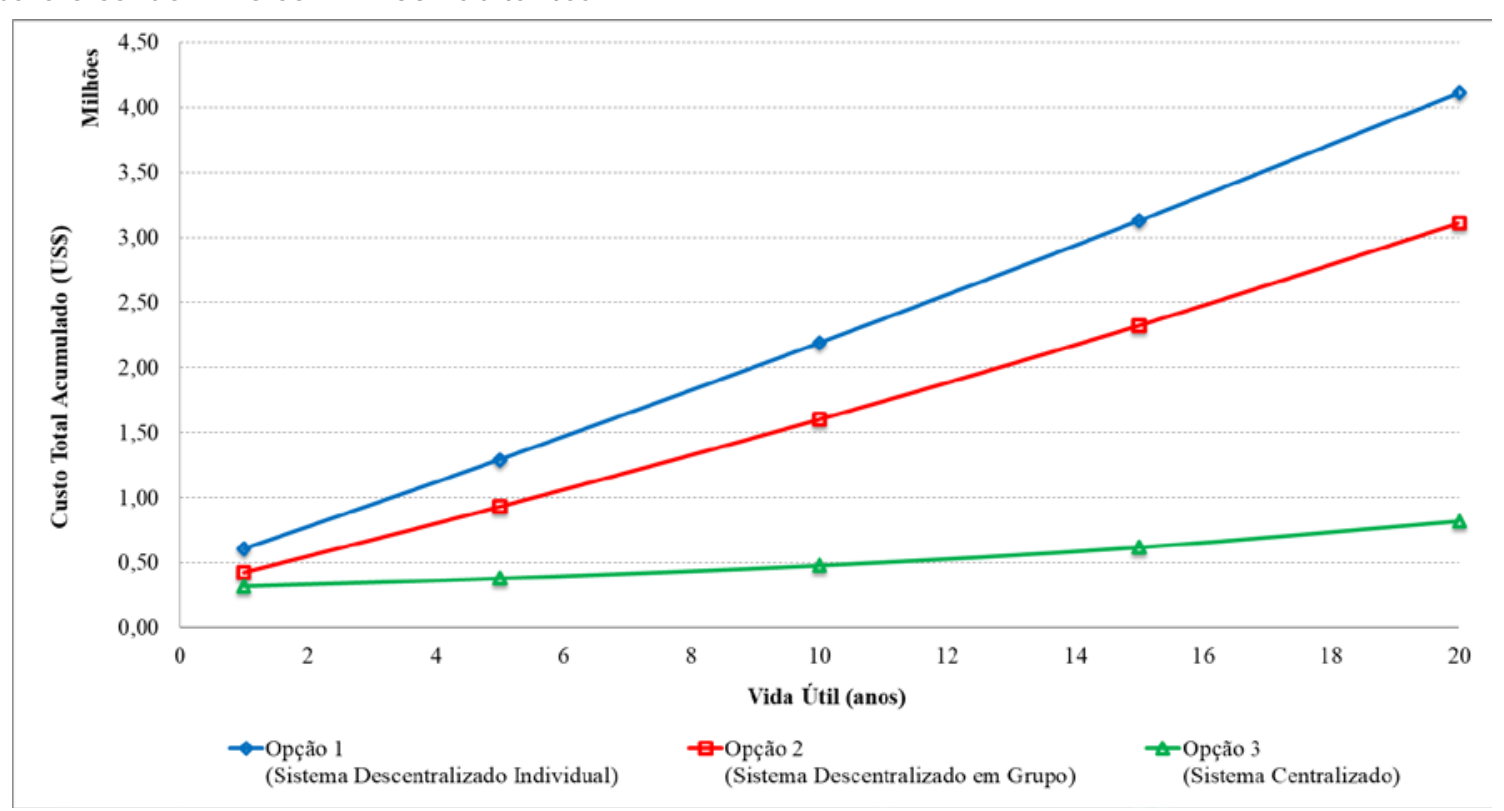

A partir da Figura 6, pode ser evidenciada a diferença entre os custos totais acumulados das três opções disponíveis. Percebe-se que a opção 1, com dez sistemas descentralizados individuais, apresenta o maior custo total. Além disso, verifica-se que vantagem da opção 3, composta do sistema centralizado, deve-se principalmente ao fato de seu custo de manutenção anual ser bastante inferior ao das opções 1 e 2 .

Considerando-se agora o efeito escala nos custos de manutenção, verifica-se ainda que a opção 3, que consiste na implantação de sistema centralizado com o tratamento por meio de reator UASB combinado com filtro biológico aerado submerso, mantém o menor custo total durante a vida útil das opções comparadas. Na Figura 7 está apresentado o custo total acumulado ao longo da vida útil de cada opção, considerando o efeito escala nas opções 1 e 2 . Os valores foram obtidos por meio do software LINDO.

Com base na Figura 7, observa-se que a opção 3, sistema centralizado, não sofreu alteração, mesmo com a considerável redução nos custos das outras duas opções, mantendo-se ainda a mais viável economicamente. Entretanto, com a inserção do efeito escala, verifica-se a inversão de vantagem entre as opções 1 e 2 . A partir do décimo segundo ano de operação, a opção 2, com cinco sistemas descentralizados em grupo, que tinha o segundo menor custo total, passa a ser a opção menos viável economicamente. Em contrapartida, a opção 1, com dez sistemas descentralizados individuais, inicialmente a mais cara, passa a ter o segundo menor custo total acumulado, devido principalmente ao fato de o sistema DBR não consumir energia elétrica durante o processo de tratamento de água residuária. 
Figura 7 - Custo total acumulado com o efeito escala durante a vida útil das opções disponíveis para o condomínio com 1.700 habitantes

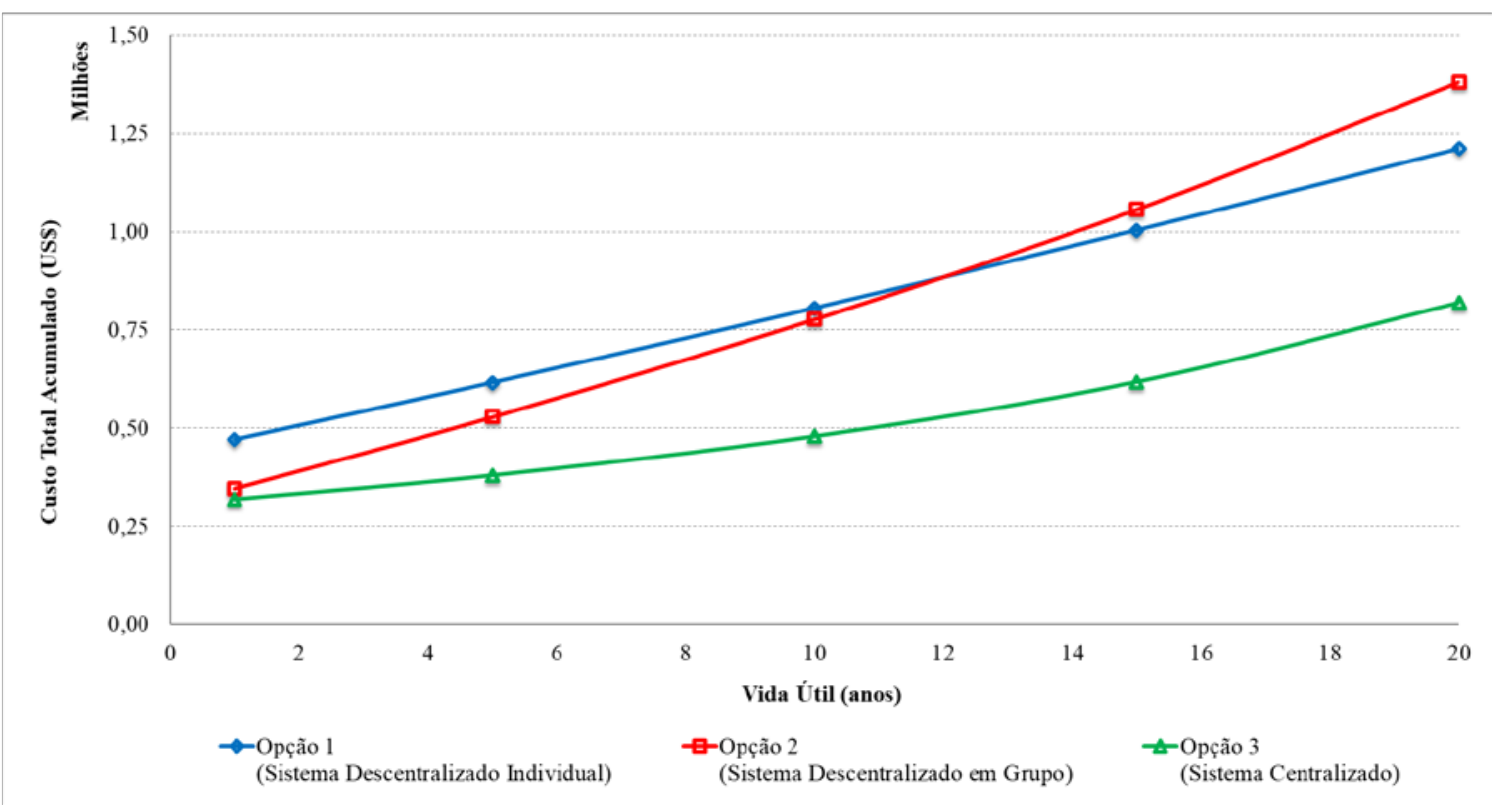

Assim, com esses resultados do modelo de PI, com e sem o efeito escala, pode-se afirmar que o sistema centralizado é a opção mais viável, o que está coerente com os trabalhos publicados na literatura, como Oliveira, Correa e Santos (2013), Guo e Englehardt (2015) e Libralato, Ghirardini e Avezzù (2012). Além disso, os resultados, com e sem o efeito escala, considerando uma vida útil de 20 anos, também confirmam o sistema centralizado como a melhor opção, embora essas bibliografias não contemplem a vida útil. Observase também que esse modelo permite analisar, entre os sistemas descentralizados, qual o mais viável para uma vida útil estabelecida.

\section{Conclusões}

Com base nos dados disponíveis na literatura e nos princípios da PI, foi possível apresentar a formulação de um modelo matemático para a tomada de decisão quanto ao tipo de sistema descentralizado individual, descentralizado em grupo ou centralizado. A vantagem desse modelo é que ele fornece o menor custo total acumulado, o custo ao longo da vida útil e a quantidade de sistemas necessários para atender a uma demanda específica.

No condomínio hipotético considerado neste trabalho constatou-se, por meio do modelo, que a instalação de uma unidade de sistema centralizado é mais viável do que cinco unidades de sistemas descentralizados em grupo e dez unidades de sistema descentralizado individual no que diz respeito aos custos de implantação, de operação e de manutenção para um período de 20 anos.

Os resultados mostram a importância de se analisar o comportamento dos custos ao longo da vida útil dos sistemas e a relevância das variáveis inseridas no modelo, porque um tipo de tratamento ou de sistema, inicialmente competitivo, pode ter o custo total acumulado superior ao das outras opções disponíveis.

Além disso, pode-se perceber que a frequência de manutenção e o efeito escala, resultante do aumento da população e da quantidade de sistemas implantados, são fatores que interferem diretamente na viabilidade de implantação dos sistemas. Observa-se que o modelo pode ser aprimorado, caso sejam consideradas diferentes frequências de manutenção para cada tipo de sistema de tratamento.

Finalmente, é importante ressaltar que esse tipo de modelo para a tomada de decisão contribui para que os profissionais façam suas análises e proponham as melhores soluções, com a possibilidade de considerar todas as fases do ciclo de vida do sistema, e não somente a fase de implantação. Outro ponto relevante é que o modelo permite a flexibilidade de análise para qualquer período do ciclo de vida dos sistemas. 


\section{Referências}

AFFERDEN, M. V. et al. A step towards decentralized wastewater management in the Lower Jordan Rift Valley. Water Science \& Technology, v. 61, n. 12, p. 181-192, 2010.

ARENALES, M. et al. Pesquisa operacional para cursos de engenharia. 8. ed. Rio de Janeiro: Elsevier, 2007.

ASANO, T. et al. Water reuse: issues, technologies, and applications. New York: McGraw Hill, 2007.

ASSOCIAÇÃO BRASILEIRA DE NORMAS TÉCNICAS. NBR 15575: edificações habitacionais: desempenho: parte 1: requisitos gerais. Rio de Janeiro, 2013.

BROSTEL, R. C.; HARADA, A. L.Análise da descentralização de sistemas de tratamento de esgotos: uma aplicação para as condições do Distrito Federal. In: CONGRESSO BRASILEIRO DE ENGENHARIA SANITÁRIA E AMBIENTAL, 19., Foz do Iguaçu, 1997. Anais [...] Foz do Iguaçu, 1997.

CASTILHO, C. P.; OLIVEIRA, L. H. Avaliação durante operação de sistemas de água não potável em edifícios residenciais. Ambiente Construído, Porto Alegre, v. 18, n. 1, p. 403-415, jan./mar. 2018.

COHIM, E. et al. Perspectivas futuras: água, energia e nutrientes. In: GONÇALVES, R. F. (org.). PROSAB 5: uso racional de água e energia: conservação de água e energia em sistemas prediais e públicos de abastecimento de água. Programa de Pesquisa de Saneamento Básico, 2009.

COLIN, E. C. Pesquisa operacional: 170 aplicações em estratégia, finanças, logística, produção, marketing e vendas. Rio de Janeiro: LTC, 2007.

COOK, S.; SHARMAA, A. K.; GURUNG, T. R. Evaluation of alternative water sources for commercial buildings: a case study in Brisbane, Australia. Resources, Conservation and Recycling, v. 89, p. 86-93, 2014.

DELL’UOMO, F. Análise técnica e econômica dos processos de tratamento de águas cinzas visando ao reuso. Rio de Janeiro, 2014. 110 f. Dissertação (Mestrado em Engenharia Ambiental) - Escola Politécnica \& Escola de Química, Universidade Federal do Rio de Janeiro, Rio de Janeiro, 2014.

DIAS, C. M. Modelos para a tomada de decisão quanto ao tipo de sistema predial de água não potável. São Paulo, 2017. 221 f. Dissertação (Mestrado em Engenharia Civil) - Escola Politécnica, Universidade de São Paulo, São Paulo, 2017.

DIXON, A. M.; BUTLER, D.; FEWKES, A. Guidelines for greywater re-use: health issues. Journal Chartered Institution of Water and Environmental Management, v. 13, p. 322-326, 1999.

DOMÈNECH, L.; SAURÌ, D. Socio-technical transitions in water scarcity contexts: public acceptance of greywater reuse technologies in the Metropolitan Area of Barcelona. Resources, Conservation and Recycling, v. 55, p. 53-62, 2010.

DOMINGUES, S. F. et al. Sistemas de aproveitamento de águas cinzas e pluviais em empreendimentos residenciais: estudo de caso. São Paulo, 2016. 25 f. Trabalho da disciplina de Desempenho e Inovação de Sistemas Prediais Hidráulicos - Escola Politécnica, Universidade de São Paulo, São Paulo, 2016.

ENGIN, G. O.; DEMIR, I. Cost analysis of alternative methods for wastewater handling in small communities. Journal of Environmental Management, v. 79, n. 4, p. 357-363, 2006.

FERREIRA, T. de V. G.; OLIVEIRA, L. H. de. Sistema descentralizado individual de água não potável: a necessidade da gestão da qualidade e da quantidade. Ambiente Construído, Porto Alegre, v. 18, n. 1, p. 373-386, jan./mar. 2018.

GARFINKEL, R. S.; NEMHAUSER, G. L. Integer programming. New York: John Wiley \& Sons, 1972.

GOLDBARG, M. C.; LUNA, H. P. L. Otimização combinatória e programação linear: modelos e algoritmos. 2. ed. Rio de Janeiro: Elsevier, 2005.

GUEST, J. S. et al. A new planning and design paradigm to achieve sustainable resource recovery from wastewater. Environmental Science \& Technology, v. 43, p. 6126-6130, 2009.

GUO, T.; ENGLEHARDT, J. D. Principles for scaling of distributed direct potable water reuse systems: a modeling study. Water Research, v. 75, p. 146-163, 2015. 
HENDRICKSON, T. P. et al. Life-cycle energy and greenhouse gas emissions of a building-scale wastewater treatment and nonpotable reuse system. Environmental Science \& Technology, v. 49, p. 10303-10311, 2015.

LIBRALATO, G.; GHIRARDINI, A. V.; AVEZZÙ, F. To centralise or to decentralise: an overview of the most recent trends in wastewater treatment management. Journal of Environmental Management, v. 94, p. 61-68, 2012.

MANOUCHEHRI, M.; KAGARI, A. Water recovery from laundry wastewater by the cross flow microfiltration process: a strategy for water recycling in residential buildings. Journal of Cleaner Production, v. 168, p. 227-238, 2017.

MARINS, F. A. S. Introdução à pesquisa operacional. São Paulo: Cultura Acadêmica; Universidade Estadual Paulista; Pró-Reitoria de Graduação, 2011.

MARQUES, I. G.; OLIVEIRA, L. H. Padronização de terminologia e de conceitos de sistemas prediais de água não potável. 2014. Disponível em: http://www.cbcs.org.br/website/comunicacaoecnica/show.asp?ppsCode=F0B31ADA-B27B-41B7-B628-AB5AF89EA5BA. Acesso em: 5 jan. 2016.

OLIVEIRA, L. H.; CORREA, F. R.; SANTOS, M. O. Conceptual model for decision-making between centralized and decentralized non-potable water systems. In: CIB W62 SYMPOSIUM WATER SUPPLY AND DRAINAGE FOR BUILDINGS, Nagano, 2013. Proceedings [...] Nagano, 2013.

OLIVEIRA, S. V. W. B. Modelo para tomada de decisão na escolha de sistema de tratamento de esgoto sanitário. São Paulo, 2004. 197 f. Tese (Doutorado em Administração) - Faculdade de Economia e Contabilidade, Universidade de São Paulo, São Paulo, 2004.

PIVELI, R. P. Apostila para tratamento de esgotos. 2004. 71 f. Apostila para disciplina de graduação. Departamento de Engenharia Hidráulica e Ambiental, Universidade de São Paulo, São Paulo, 2004.

REAMI, L.; CORAUCCI FILHO, B. Estimativas de custos unitários de implantação de estações de tratamento de esgotos. 2010. Disponível em:

http://www.fenasan.com.br/arquivos/2015/09h40_luciano_reami_06-08_sant3.pdf. Acesso em: 5 jan. 2016.

SCHEE, W. G. van der. Experiences with a collective domestic water system in Leidsche Rijn. In: CIB W62 SYMPOSIUM WATER SUPPLY AND DRAINAGE FOR BUILDINGS, Paris, 2004. Proceedings [...] Paris, 2004.

UNITED STATES ENVIRONMENTAL PROTECTION AGENCY. Primer for municipal wastewater treatment. Washington: United States Environmental Protection Agency, EPA 832-R-04-001, 2004. Disponível em: http://www3.epa.gov/npdes/pubs/primer.pdf. Acesso em: 15 nov. 2015.

VON SPERLING, M. Introdução à qualidade das águas e ao tratamento de esgotos. 3. ed. Belo Horizonte: Departamento de Engenharia Sanitária e Ambiental da Universidade Federal de Minas Gerais, 2005. 


\section{Christiane Miranda Dias}

Departamento de Engenharia de Construção Civil, Escola Politécnica | Universidade de São Paulo| Av. Prof. Almeida Prado n. 83, Trav. 2 Cidade Universitária | São Paulo - SP - Brasil | CEP 05508-070 | Tel.: (11) 3091-5458 | E-mail: christinemdias@gmail.com

\section{Lúcia helena de Oliveira}

Departamento de Engenharia de Construção Civil, Escola Politécnica | Universidade de São Paulo | E-mail: lucia.helena@ usp.br

\section{Fernando Akira Kurokawa}

Departamento de Engenharia de Construção Civil, Escola Politécnica | Universidade de São Paulo | Tel.: (11) 2648-6537| E-mail: fernando.kurokawa@usp.br

\section{Ambiente Construído}

Revista da Associação Nacional de Tecnologia do Ambiente Construído

Av. Osvaldo Aranha, 99 - 3o andar, Centro

Porto Alegre - RS - Brasil

CEP $90035-190$

Telefone: +55 (51) 3308-4084

Fax: +55 (51) 3308-4054

www. seer. ufrgs. br/ ambienteconstruido

E-mail: ambienteconstruido@ufrgs.br

(c) () 